\title{
Proper Hand Washing for Food Handlers
}

Hand washing is one of the primary methods used to help prevent foodborne illness. Proper hand washing will reduce your risk of transmitting disease-causing microorganisms such as bacteria, viruses, and other agents to people who eat the food you handle. If you need to use hand sanitizer, always wash your hands first! Dirt, foods, or anything else on your hands makes the alcohol less effective. Do not use hand sanitizer in place of hand washing!
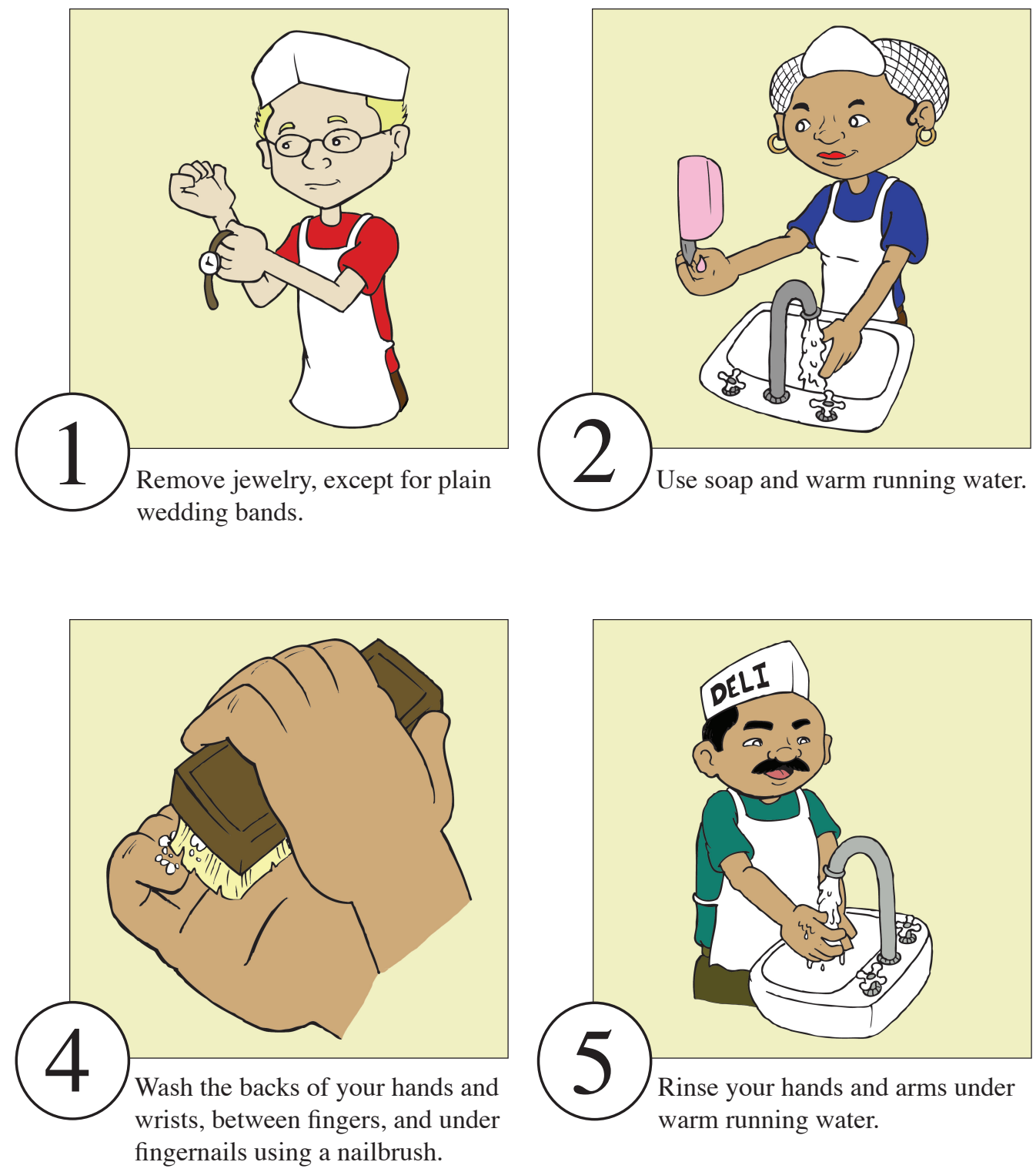

wrists, between fingers, and unc
Wash your hands before:

- Entering a food service station

- Handling ready-to-eat or raw foods

- Touching serving utensils

- Putting on new gloves

- Handling different foods

- Serving foods

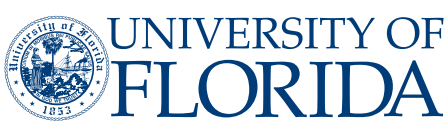

IFAS EXTENSION
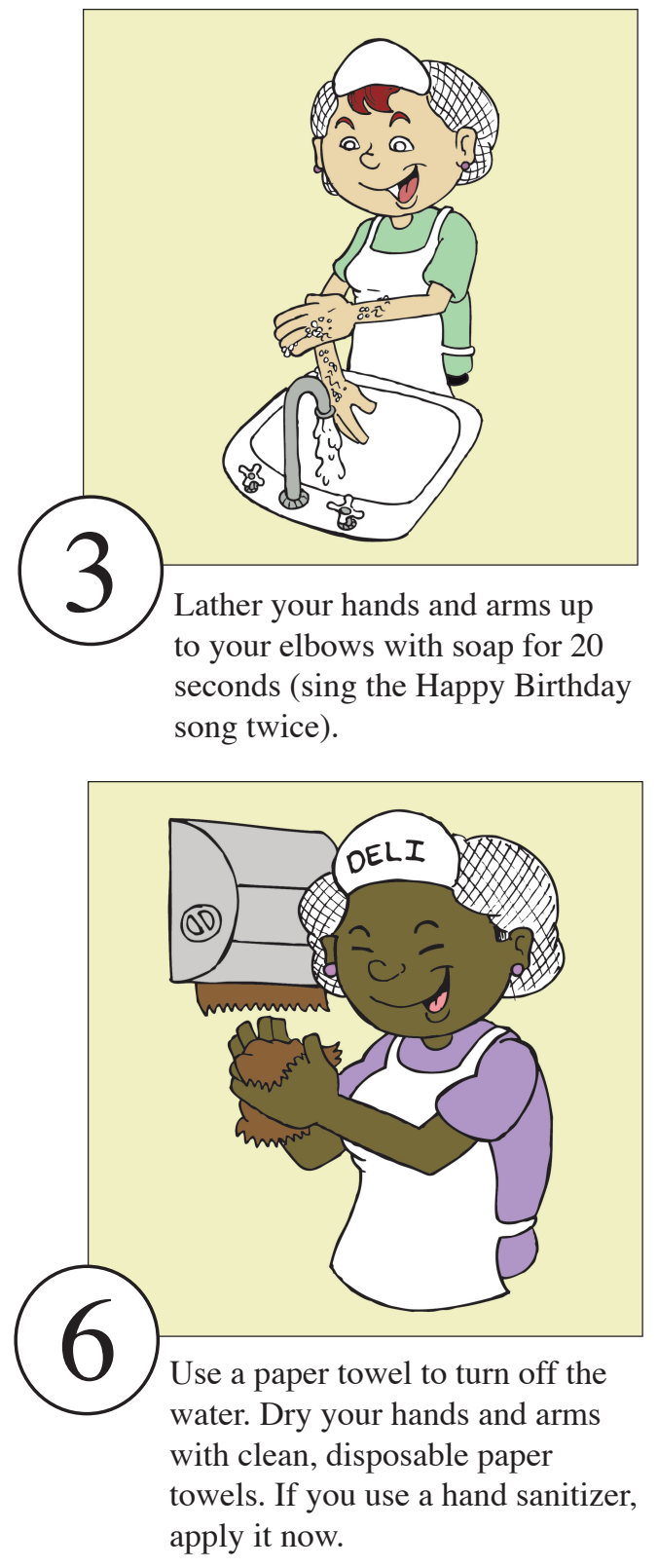

\section{Wash your hands after:}

- Visiting the restroom

- Touching bare human body parts (ears, nose, hair, etc.)

- Handling garbage

- Working with raw foods

- Handling cleaning chemicals

- Doing other activities that dirty your hands 